\section{Superconductivity to cosmology: K. Alexander Müller explores mysteries in energy}

Interviewed by Anke Weidenkaff

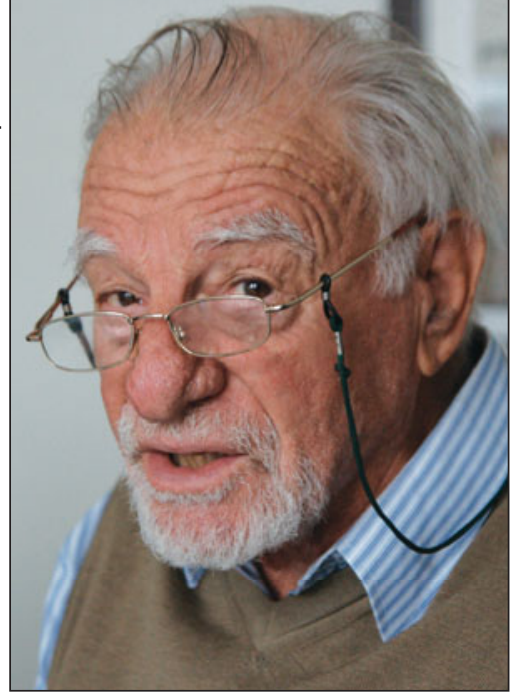

In 1913, physicist Heike Kamerlingh-Onnes received the Nobel Prize for liquifying He and his discovery of superconductivity two years prior. It would be over 76 years later until K. Alexander Müller, together with Johannes Georg Bednorz, would be honored as Nobel laureates for their discovery of high-temperature superconductivity (HTS), grounded in their research with metal oxides. When we asked Müller, recently, what he would advise young materials scientists in regards to research for energy, he said, "I've always been a fan of oxides, therefore to work in oxide would not be bad."

MRS BULLETIN: What motivated you to work on materials for superconductivity?

K. ALEXANDER MÜLLER: Over the years I did various studies with oxides such as structural phase transitions, ferroelectricity, and photochromic effects. I detected electron paramagnetic resonance of high valent transition metal ions. But I always wondered, what about superconductivity? This was one part. The other part was that IBM had a huge project to generate a computer with superconductivity.

At IBM, we formed a group of four people. We started with page one of Michael Tinkham's book Introduction to Superconductivity, and every two or three days, we read two pages, then got together to discuss it. After some work, we came to understand how superconductivity resulted from percolation in granular $\mathrm{Al}$, and we decided to submit a paper to Physical Review Letters, but management felt this was an exposure. I had to fight for about six weeks until I got permission to publish.

Oxides were just dirty materials. But nowadays they replace silicon because of a huge effort of the com- munity to get it pure and crystalline.

IBM had put a large effort in this superconductivity program, but they eventually closed it down.

\section{How did your discovery of HTS} change today's energy supply chain? First we had mechanical means to apply energy, such as windmills in Holland. The second major innovation was when Werner von Siemens invented the dynamo. Now we could make generators and motors and transport energy of a few hundred kilometers by alternating current. And now we have this debate about the high costs of windmills in the North Sea. Companies in Germany went bankrupt because you have to rebuild a new grid network. This is costly, but we are rich enough, and in the end if it does not work, we might buy nuclear energy from Poland and France.

Now we can turn our attention to generate electric power from the sun via solar panels. Sooner or later, this is going to come, which means we'll have to transport this electrical energy over 3000-4000 kilometers whether in China, Europe, or the United States. How to transport electrical energy is being studied intensively. China has already decided how to do it with superconducting copper oxides, because they have large amounts of the rare earths needed. In the US and Europe, the alternative magnesium diboride is discussed instead, which has a transition of $50 \mathrm{~K}$, so you have to cool it with hydrogen, which is a safety question. A leak would probably lead to an explosion, so you have to see. Long distance transport has to be DC, because the losses for AC are too large.

After receiving the Nobel Prize, what was it like?

I was able to capitalize on this honor by helping to advance scientific projects in Switzerland. In particular, I was asked to be in charge of the committee to establish a Swiss Light Source [SLS]. We held a three-day workshop. On the first day, we considered a large facility for cyclotrons that we were planning; the second day, we addressed the science for synchrotron radiation; and the third, we had a political discussion. We emphasized the importance of this Swiss Light Source not only for Zürich, but for the whole area of south Germany. The nice thing about this SLS was that it was finished in time, within the budget. SINQ neutrons are complementary. Based on these successes, now the free-electron laser will be realized. So, I did something good for Switzerland and paid back.

\section{Following the discovery of HTS,} how did you deal with the very high expectations for materials?

In his book The Privilege of Being $a$ Physicist, Victor Weisskopf touches on many things, from nuclear weapons to energy production. And he asked, "How long does it take from the moment you see a physics effect until it's used?" I started looking for the shortest and longest examples. The shortest is X-rays/Röntgen. Within half a year, we were looking at broken bones and using x-rays in the First World War to 
look at injuries. Even Madame Curie herself was running such an x-ray station behind the Western Front. Fuel cells were found 160 years ago.

So, a quarter of a century ago, an effect was found, and by now we have two branches. One is the small current application where we can make circuits with very sharp, high-Qs, and from that we can make filters, which are only square. They also are partially used for these small currents and for data logic; this is what [Jochen] Mannhart was doing in Augsburg.

To lead the current, we use copper oxides because the conductivity of heat is much lower than for intermetallic compounds. I think CERN was probably one of the first who really used it. When I was at CERN, there was a cooperative meeting of the application of superconductivity with neodymium titanium coils, but this high-energy physics wants to go to higher and higher energies. Before shooting it down, it was $7 \mathrm{TeV}$, and after redoing it, they went to twice as much at $14 \mathrm{TeV}$. It's probably CERN who will reach the highest possible energy, where they are still looking for dark matter.

\section{What do you consider the fore- most future development in energy research?}

In the medium-time schedule, we may have nuclear power plants in the Swiss
Mountains that are quite safe. However, I think it was not such a good idea to put them into areas where you have the strongest density of population. To put them underneath in the mountains may be engineer-wise, not a big question. If we are able to drill a hole of $57 \mathrm{~km}$ through the Alps, it should be possible to drill $20 \mathrm{~km}$ of a hole to a place that is safe. Of course, you have to invest money. Japan located their power plants at Fukushima on the Pacific side where you have strong waves. It was done because they could save on copper wires to bring the energy to the place where they use it. If they would put the plant on the side of the Strait of Tsushima toward China, they would have to extend their wires for $200 \mathrm{~km}$ or $300 \mathrm{~km}$ more, which we do all the time in Switzerland when we bring energy from the water station to here.

\section{If you could start your research career now, what directions would you choose?}

If I would be a little better, say, in mathematics, I would probably embark on cosmology. By gravitation, 80-90\% of the universe is filled with invisible particles. Laura Baudis, a professor at the University of Zürich, has a huge pot of liquid xenon, surrounded with scintillation counters. She hopes that if such a particle traverses, makes a spark, and they detect something - but they

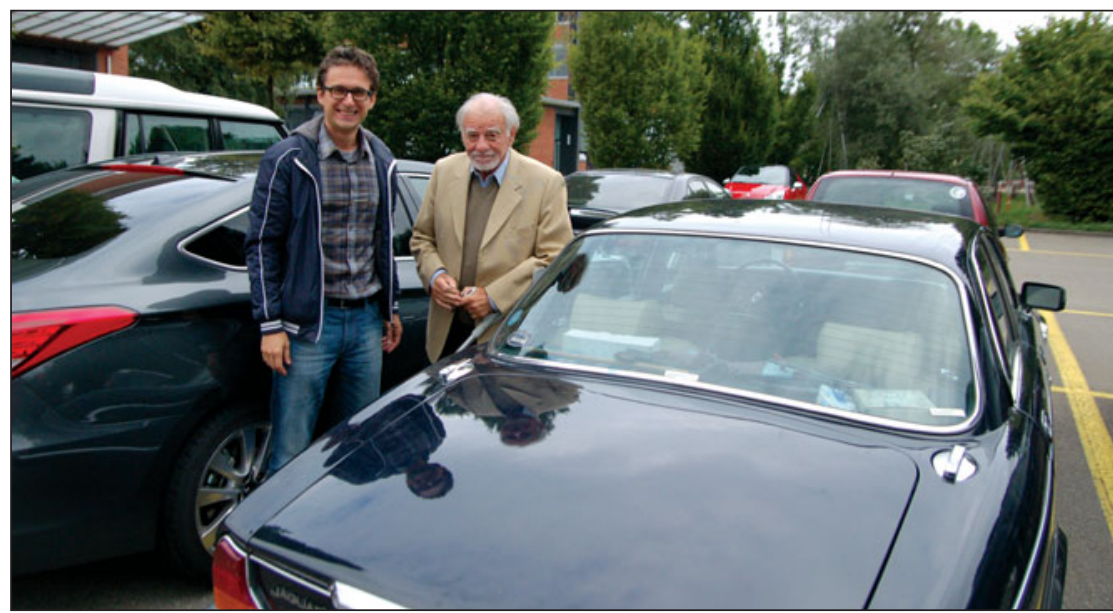

K. Alexander Müller (right) with Andrey Shkabko.

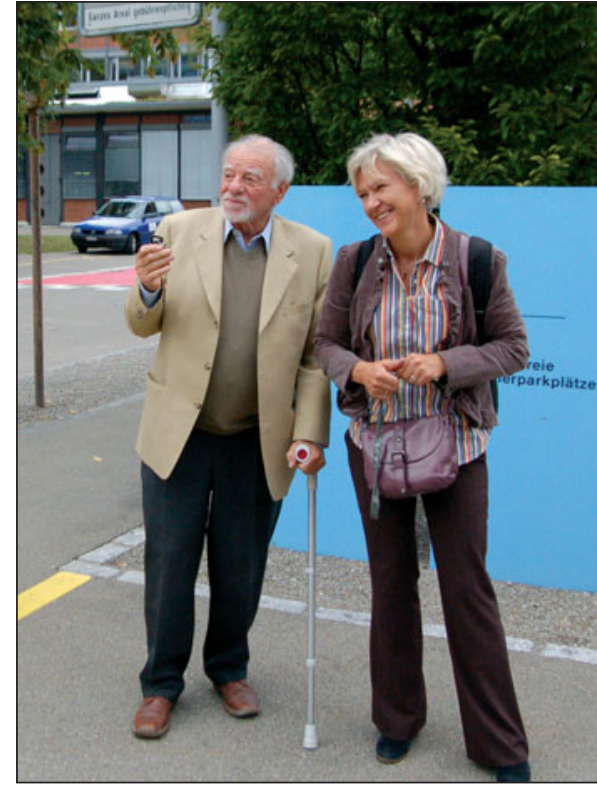

K. Alexander Müller with Anke Weidenkaff

don't see anything. A group from the University of Munich has a monocrystal of calcium wolframate $\mathrm{CaWO}_{4}$. On top of it they have a thin layer of tungsten, which superconducts at, perhaps, 0.0 and something Kelvin. They measure the current through this layer, hoping that when a particle goes through, it makes a spike, but ... nothing.... With the total energy of $7 \mathrm{TeV}$, CERN gets huge amounts of particles that they can detect when they smash protons on each other, saying officially, "We are looking for the Higgs Boson." But they're also looking for dark matter and dark energy, but see nothing. The theoreticians, as far as I understand, presuppose that the interaction is there based on what we see, the visible ones. There are four interactions: electromagnetic, gravitation, and weak and strong nuclear forces. I asked a colleague, "Hey, are you sure that in what you don't see, you have also all four interactions?" We are sure we have gravitation because they see deviations for orbits. Regarding the other three, he said, "If these groups from Zürich and Munich don't see anything, it means they don't see any scintillation, which means they don't see any electromagnetic radiation. So maybe this space has no electromagnetic force but has something else, which we don't know." And this is of course very interesting, amazing. 


\section{Advancing \\ Materials Characterization}

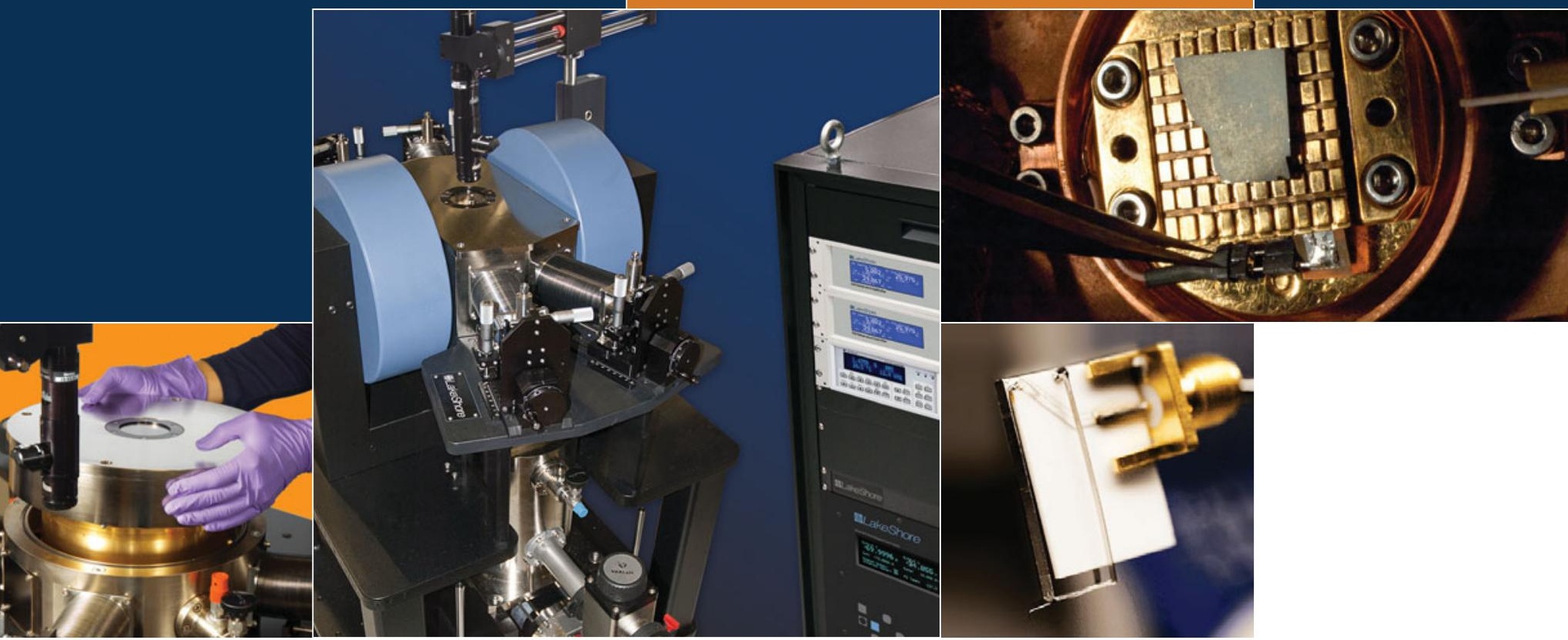

Flexible and Expandable Probing

- Up to 6 micro-manipulated probe arms

- Thermal anchoring minimizes sample heating

- DC/RF probing to $1 \mathrm{GHz}$

- Microwave probing to $67 \mathrm{GHz}$

- Fiber optic arm modifications available

MRS Booth 301

\section{Cryogenic and Cryogen-free Probe Stations}

Lake Shore's cryogenic probe stations provide precisely controlled environments for non-destructive measurement of the electrical properties of materials and early-stage electronic devices.

Typical applications include sampling IV and CV curves over a wide range of temperatures, measuring microwave and electro-optical responses, characterizing magneto-transport properties in variable magnetic fields, Hall effect measurements to understand carrier mobility, and a variety of other material studies.

Look to Lake Shore for the expertise and technology to support your work.

\section{Lake Shore} CRYOTRONICS

ADVANCING SCIENCE 614.891.2243 | www.lakeshore.com

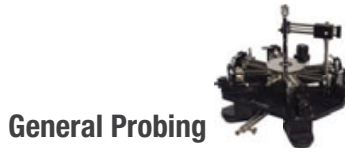

Versatile and flexible research platforms

- Tabletop and stand designs

-1.6 K to $675 \mathrm{~K}$ temperature range

- Up to 4 inch wafer probing available
Magnetic Field Probing

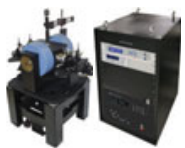

Horizontal and vertical field magnet station designs

- Vertical fields to $2.5 \mathrm{~T}$

- Horizontal fields to $1 \mathrm{~T}$

- As low as $1.9 \mathrm{~K}$ base temperature 